Original Article

\title{
Verification of spatial recognition ability of stroke patients required to resume automobile driving
}

\author{
Kouhei Todate, RPT, MS ${ }^{1,2)}$, Akiyoshi Takami, RPT, PhD²), Misato Makino, RPT, PhD ${ }^{2)}$ \\ 1) Mihono Hospital: 31-2 Oyama, Okubo, Hachinohe-shi, Aomori 031-0833, Japan \\ 2) Hirosaki University Graduate School of Health Sciences, Japan
}

\begin{abstract}
Purpose] To help patients who had a stroke resume automobile driving, we evaluated their ability to recognize three-dimensional space like that experienced in actual driving situations, with a focus on sensing car width. [Participants and Methods] Seven patients who had a stroke and 29 healthy adults participated in the study. Three 50-cm-wide chairs and 2 panels, $3 \mathrm{~m}$ apart, were placed $9 \mathrm{~m}$ from the chair in which the participant sat. One panel was fixed and the other panel could be moved toward the fixed panel in a horizontal direction. The participants were asked to signal when they believed that the width of the chair was the same as the space between the panels. [Results] In the simulation of driving a real car, the mean error in judging distance was $13.2 \pm 10.4 \mathrm{~cm}$ for the Healthy group and that of the Stroke group was two times greater than that of the Healthy group. [Conclusion] These findings show that spatial recognition cannot be evaluated using paper-based two-dimensional higher brain function tests. To help patients who had a stroke resume driving requires evaluation of three-dimensional spatial recognition ability under circumstances that simulate actual driving situations.

Key words: Driving, Stroke, Spatial recognition
\end{abstract}

(This article was submitted Aug. 10, 2020, and was accepted Mar. 17, 2021)

\section{INTRODUCTION}

Persons with brain injuries due to stroke or accident suffer from impaired physical or higher brain functions and may be forced to discontinue automobile driving. If people are not allowed to drive cars, then their participation in social activities is inhibited and furthermore, the quality of their life may be reduced. Medical institutions are sometimes asked to conduct examinations to determine if persons with brain injuries can resume driving or not. However, in reality, there are no standardized criteria for making a judgment on a patient's ability to resume driving, and each medical institution therefore has to make its own judgment.

Stroke patients who want to resume automobile driving have to take the aptitude test according to the operating standards of the Japanese National Police Agency and on the basis of the test results, it is judged if they can resume driving or not. Higher brain function impairment follows the rules related to "dementia" and many studies have already reported the use of paper-based examinations for higher brain functions to make a judgment on whether or not stroke patients can resume driving ${ }^{1-4)}$. However, even when stroke patients are allowed to resume driving on the basis of aptitude test results, a portion of these patients have issues during actual driving, which may indicate that the two-dimensional evaluation cannot adequately serve as an examination of the ability to recognize three-dimensional space. Although studies on the use of driving simulators $^{5,6)}$ and actual cars ${ }^{7-10)}$ to determine the ability to resume driving were reported, these simulators are quite expensive and there are limited locations available for the assessment with actual cars.

In light of the above, the authors have become aware of the need for testing with which any medical institution can

*Corresponding author. Akiyoshi Takami (E-mail: a-takami@hirosaki-u.ac.jp)

(C2021 The Society of Physical Therapy Science. Published by IPEC Inc.

(c) (1) $\odot$ This is an open-access article distributed under the terms of the Creative Commons Attribution Non-Commercial No Deriva-

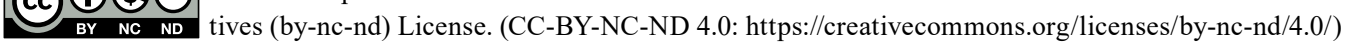


easily evaluate the ability to recognize three-dimensional space closer to the actual situation, rather than paper-based twodimensional testing, in order for stroke patients to be allowed to resume automobile driving. One of the important factors in recognizing three-dimensional space is spatial recognition ability - that is, the ability to understand how objects occupy a three-dimensional space and their relationship with each other, in terms of understanding the location and direction of an object as well as its distance from other objects. Spatial recognition ability is the somatic sensation required for automobile driving. No previous studies have investigated application of the evaluation of spatial recognition ability to assessments for the resumption of automobile driving. There are no standardized methods for verification of spatial recognition ability and at present, no standard values for spatial recognition ability for not only stroke patients but also healthy adults. Therefore, in this this study, the authors paid special attention to spatial recognition ability, which is a physical function required for supporting stroke patients in the resumption of automobile driving, and carried out investigations for the following purposes. One is to focus on sensations of car width which is one factor in the ability to gauge spatial recognition ability. The other is to compare stroke patients with healthy adults as a basic study for creating reference values.

\section{PARTICIPANTS AND METHODS}

The participants are described as follows. Seven patients who had suffered from stroke and had been receiving physical therapy as in-patients in the recovery-phase rehabilitation ward of our hospital were included in the study (Stroke group). These patients met the criterion that they had driven cars just before the onset of stroke and in addition, the inclusion and exclusion criteria reported by Katayama ${ }^{11)}$ were used to select the study participants (Table 1). A control group consisted of twenty-nine healthy adults who were driving habitually (Healthy group). This study was conducted with the approval of the Ethics Committee at Hirosaki University Graduate School of Health Sciences (Reference No.: 2016-045) and of the Ethics Committee of Total Rehabilitation Mihono Hospital (Acceptance No.: 7). Informed consent to participate in this study was obtained in writing from each participant who had been fully informed of the objectives and methods of this study.

The method is described below. As demographic factors common to the two groups, information on gender, age, duration of holding drivers' licenses, and frequency of driving was collected. For the Stroke group, the injured hemisphere, length of time since onset, and presence or absence of sensory disturbance were identified through a medical interview and medical records (Table 2). The participants in the Stroke group were tested on higher brain functions based on the evaluation of driving ability: (1) line bisection test; (2) Mini-Mental State Examination (MMSE); (3) Trail Making Test A and B (TMT); and (4) Kana [Japanese character] pick-out test.

Spatial recognition ability was determined using three chairs of $50 \mathrm{~cm}$ each in width. The widths were set at $50 \mathrm{~cm}$, $100 \mathrm{~cm}$, and $150 \mathrm{~cm}$ for the following reasons: $150 \mathrm{~cm}$ was selected since it is a car width common to Japan-made subconpact cars or compact cars; $50 \mathrm{~cm}$ is close to the common body width of Japanese people when viewed from the front; and $100 \mathrm{~cm}$ was in the middle of the above. The width was adjusted by changing the number of chairs used. Under each of these conditions, a participant sat on one chair while facing the front. Two panels were deployed $9 \mathrm{~m}$ away from the chair and at an interval of $3 \mathrm{~m}$ between the two. One panel was fixed and the other was moveable, where the moveable panel could be moved toward the fixed panel in a horizontal direction manually $(0.1 \mathrm{~m} / \mathrm{sec})$. The participant was asked to give a signal when he/she judged that the width of the chair was the same as the interval of the panels. When the signal was given, the distance between

Table 1. Inclusion and exclusion criteria

\begin{tabular}{l}
\hline Inclusion criteria \\
\hline 1) Hospitalized due to stroke \\
2) Vital signs are stable \\
3) Consciousness is lucid \\
4) With driving license, driving history \\
5) Locomotion in a ward is independent \\
\hline Exclusion criteria \\
1) Diplopia, visual impairment \\
2) With aphasia, unilateral spatial neglect \\
3) Have a history of seizures
\end{tabular}

Table 2. Baseline characteristics of stroke group and healthy group

\begin{tabular}{lcc}
\hline & $\begin{array}{c}\text { Stroke group } \\
(\mathrm{n}=7)\end{array}$ & $\begin{array}{c}\text { Healty group } \\
(\mathrm{n}=29)\end{array}$ \\
\hline Age (years, mean \pm SD) & $61.7 \pm 9.3$ & $25.7 \pm 3.0$ \\
Gender, n (\%) & $6(85.7)$ & $17(58.6)$ \\
$\quad$ Male & $1(14.3)$ & $12(41.4)$ \\
Female & $468.0 \pm 117.0$ & $85.2 \pm 35.2$ \\
Duration of holding drivers' licenses (months) & \\
Driving frequency, n (\%) & $7(100)$ & $1(3.4)$ \\
$\quad<6$ times/week & $0(0)$ & $28(96.6)$ \\
$>6$ times/week & & - \\
Injured hemisphere, $\mathrm{n}(\%)$ & $2(28.6)$ & - \\
Left & $5(71.4)$ & - \\
$\quad$ Right & $126.0 \pm 66.2$ & - \\
Length of time since onset (days) & & \\
Sensory disturbance, $\mathrm{n}(\%)$ & $7(100)$ & \\
Presence & $0(0)$ & \\
Absence & & - \\
\hline
\end{tabular}


the two panels was measured. An absolute value of the difference between the measured distance and the chair's width was regarded as an "error in spatial recognition ability".

A total of 9 measurements, i.e., 3 times each for the chair's width of $50 \mathrm{~cm}, 100 \mathrm{~cm}$, and $150 \mathrm{~cm}$, was randomly conducted, and mean values were calculated. Arrangement of the chairs allowed simulation of driving (right side) and passenger (left side) seats., the left end of the fixed panel was placed above a line extending from the right edge of the chair, and the moving panel was placed on the left side of the participant's field of vision. The width of chair(s) was adjusted by changing the number of chair(s) added to the left side of the chair in which the participant was seated. For the passenger seat, the reverse set-up was used compared to that of the driving seat. For statistical analysis, the Mann-Whitney test was used for a comparison between the Healthy group and the Stroke group on the results obtained for each test condition under the simulated driving and passenger seats. The statistical software used was R2.8.1 (CRAN). The significance level was 5\%.

\section{RESULTS}

Table 3 shows the results of examinations on higher brain functions in the Stroke group. MMSE and screening for unilateral spatial neglect, both of which are also used to examine mental status and screen out those with dementia, did not reveal findings suggestive of extreme neglect or cognitive function impairment. TMT and the Kana pick-out test, which provide indications for attention impairment, revealed individual variations in the test results.

Table 4 shows the results for errors in spatial recognition ability. Statistical analysis revealed that under the conditions of the driving seat and the chair's width of $150 \mathrm{~cm}$, the error was significantly greater in the Stroke group than in the Healthy group $(\mathrm{p}<0.01)$. The same tendency was also noted with the condition of the chair's width of $50 \mathrm{~cm}$, although no significant difference was noted. Under the condition of the passenger seat, no significant difference was found between the Stroke group and the Healthy group for any of the chair's widths, although there was a tendency for the Stroke group to give greater errors.

\section{DISCUSSION}

Under the circumstances simulating the driving seat of a real car (i.e., under the conditions of the simulated driving seat and the chair's width of $150 \mathrm{~cm}$ ), the mean error in spatial recognition ability to recognize the object located $9 \mathrm{~m}$ apart from the participants was $13.2 \pm 10.4 \mathrm{~cm}$ for the Healthy group. This remained within $10 \%$ of the width of the chair in which the participant was seated. In contrast, it exceeded $10 \%$ under the condition of the passenger seat, suggesting that spatial recognition ability may be acquired by habitual sitting conditions. For the Stroke group, the same parameter gave a mean of $31.3 \pm 20.0 \mathrm{~cm}$, which was two times greater than that of the Healthy group. Makishita et al. ${ }^{12)}$ reported that the accuracy of

Table 3. Results of examinations on higher brain functions in the stroke group

\begin{tabular}{lrrrrrrr}
\hline & No. 1 & No. 2 & No. 3 & No. 4 & No. 5 & No. 6 & No. 7 \\
\hline Line bisection test $(\mathrm{mm})^{*}{ }^{1}$ & -10 & 5 & 6 & 12 & 3 & -4 & -2 \\
MMSE score & 30 & 30 & 30 & 24 & 23 & 28 & 28 \\
TMT-A (sec) & 143 & 77 & 94 & 144 & 185 & 166 & 150 \\
TMT-B (sec) & 174 & 130 & 134 & 177 & 514 & 219 & 164 \\
Kana pick-out test (\%) & 100 & 100 & 100 & 82.9 & 28.6 & 65.6 & 55 \\
\hline
\end{tabular}

MMSE: Mini Mental State Examination; TMT: Trail Making Test.

${ }^{* 1}$ Shift from the midpoint of the line to the left: +, to the right: -

Table 4. Results of errors in spatial recognition ability

\begin{tabular}{|c|c|c|c|c|}
\hline & \multicolumn{2}{|c|}{ Simulation of driving seats } & \multicolumn{2}{|c|}{ Simulation of passenger seats } \\
\hline & $\begin{array}{c}\text { Stroke group } \\
(n=7)\end{array}$ & $\begin{array}{c}\text { Healty group } \\
(n=29)\end{array}$ & $\begin{array}{l}\text { Stroke group } \\
(\mathrm{n}=7)\end{array}$ & $\begin{array}{l}\text { Healty group } \\
(\mathrm{n}=29)\end{array}$ \\
\hline $50 \mathrm{~cm}$ & $\begin{array}{c}18.3[12.3,19.5] \\
19.7 \pm 15.5\end{array}$ & $\begin{array}{c}9.3[5.6,16.7] \\
11.0 \pm 6.4\end{array}$ & $\begin{array}{c}8.0[7.3,23.0] \\
20.0 \pm 22.1\end{array}$ & $\begin{array}{c}8.3[5.6,16.3] \\
11.9 \pm 10.0\end{array}$ \\
\hline $100 \mathrm{~cm}$ & $\begin{array}{c}11.0[8.7,30.5] \\
19.3 \pm 15.7\end{array}$ & $\begin{array}{c}12.3[7.3,21.3] \\
16.0 \pm 11.8\end{array}$ & $\begin{array}{c}13.6[10.5,31.3] \\
24.9 \pm 24.5\end{array}$ & $\begin{array}{c}11.6[7.6,23.3] \\
16.3 \pm 12.5\end{array}$ \\
\hline $150 \mathrm{~cm}$ & $\begin{array}{c}19.7[18.0,42.7] \\
31.3 \pm 20.0^{*}\end{array}$ & $\begin{array}{c}9.3[7.3,16.7] \\
13.2 \pm 10.4 \\
\end{array}$ & $\begin{array}{c}21.6[12.7,38.7] \\
28.7 \pm 23.6 \\
\end{array}$ & $\begin{array}{c}13.3[8.0,31.3] \\
20.0 \pm 13.4\end{array}$ \\
\hline
\end{tabular}

Median [1st quartile, 3rd quartile].

Mean \pm SD.

Mann-Whitney test. ${ }^{*} \mathrm{p}<0.05$. 
visual measurement of the inter-vehicle distance tended to increase with aging. Assuming that the spatial recognition ability associated with driving improves with aging, the results of this study show the opposite result, these results indicated that the Stroke group overestimated the distance between the objects located in the distal space, in relation to the proximal space, and therefore did not properly recognize the mutual relationship between proximal and distal spaces.

Regarding the fundamental characteristics of the participants in the Stroke group, the results of these examinations indicated that none of them suffered from significant cognitive impairment. Six of seven participants obtained MMSE scores higher than cutoff value of 24 points, but only one participant on 23 points. In addition, the line bisection test that is a screening examination for unilateral spatial neglect revealed that none of the participants had clear neglect symptoms.

Regarding studies on the prediction of driving skills based on examination results for higher brain functions in persons with brain injuries, Yamada et al. ${ }^{10)}$ reported that the cutoff value for TMT-A was 119 sec. On the basis of this previous studies, evaluation of the higher brain function examination results obtained from the participants in this study revealed that only two participants, i.e., Nos. 2 and 3, cleared the cutoff value and the standard value, whereas the other participants may have been affected by attention impairment. However, the participants were all independent, using wheelchairs or walking while they were living in the ward, and the impact of attention impairment was expected to be small on their daily living activities. Therefore, the results of the examinations for higher brain functions indicated that the participants in the Stroke group maintained general mental function, did not have neglect symptoms, and suffered from some attention disorders.

If the standard value for the error in spatial recognition ability is considered to be around $10 \%$ of the chair on which the participants sat, on the basis of the error in spatial recognition ability in the Healthy group, the error obtained for the Stroke group was far greater than that standard value. These findings show that spatial recognition ability cannot be evaluated by paper-based two-dimensional higher brain function tests and that support for patients with brain injuries in resumption of driving requires evaluation of three-dimensional spatial recognition ability under circumstances closer to actual driving situations. The greater error in spatial recognition ability in the Stroke group may be attributable to impaired physical perception which is a sequelae of stroke. Impaired physical perception includes a lack of body axis deviation ${ }^{13)}$. The neural network involving the inferior parietal lobe, the posterior part of the inferior frontal gyrus, and the thalamus play important roles in spatial attention. Injuries of the neural network cause disturbances in spatial attention. All participants in this study had supratentorial lesions: the neural network might have been impaired by lesions of the thalamus or basal ganglia, leading to impairments of physical perception and spatial attention, which then resulted in the greater error in spatial recognition ability in the Stroke group.

Corbetta et al. ${ }^{14)}$ highlighted the importance of functions of the right cerebral hemisphere in spatial attention. A study on brain hemodynamics of participants with brain injuries while driving ${ }^{15)}$ reported that significant activity was noted in the frontal lobe, parietal lobe, and temporal lobe, and that the activity was more extensive in the right cerebral hemisphere than in the left cerebral hemisphere. A study which verified the differences in mistakes while driving due to differences in the damaged cerebral hemisphere after stroke ${ }^{16)}$ reported that the frequency of mistakes was high in persons with right cerebral hemisphere damage. It is thought that there is a difference in the impact on spatial attention while driving between right and left cerebral hemisphere injuries. In this study, one of the factors contributing to the increased error in spatial recognition ability in the Stroke group may be the greater number of participants with right hemisphere injuries in this group. Further investigation is necessary to determine whether or not the error in spatial recognition ability is different between right and left hemisphere injuries.

\section{Conflict of interest}

There are no conflicts of interest to disclose.

\section{REFERENCES}

1) Kato T, Suetsuna T, Shiino M, et al.: Predictable neuropsychological test on driving skills for patients with brain disorders: clinical practice in Japan. Jpn J Occup Theapy, 2015, 49: 100-105.

2) Choi SY, Lee JS, Oh YJ: Cut-off point for the trail making test to predict unsafe driving after stroke. J Phys Ther Sci, 2016, 28: 2110-2113. [Medline] [CrossRef]

3) Choi SY, Yoo DH, Lee JS: Usefulness of the driveABLE cognitive assessment in predicting the driving risk factor of stroke patients. J Phys Ther Sci, 2015, 27: 3133-3135. [Medline] [CrossRef]

4) Mazer BL, Korner-Bitensky NA, Sofer S: Predicting ability to drive after stroke. Arch Phys Med Rehabil, 1998, 79: 743-750. [Medline] [CrossRef]

5) Hitosugi M, Takehara I, Watanabe S, et al.: Support for stroke patients in resumption of driving: patient survey and driving simulator trial. Int J Gen Med, 2011, 4: 191-195. [Medline] [CrossRef]

6) Lew HL, Poole JH, Lee EH, et al.: Predictive validity of driving-simulator assessments following traumatic brain injury: a preliminary study. Brain Inj, 2005, 19: 177-188. [Medline] [CrossRef]

7) Akinwuntan AE, DeWeerdt W, Feys H, et al.: Reliability of a road test after stroke. Arch Phys Med Rehabil, 2003, 84: 1792-1796. [Medline] [CrossRef]

8) Akinwuntan AE, De Weerdt W, Feys H, et al.: The validity of a road test after stroke. Arch Phys Med Rehabil, 2005, 86: 421-426. [Medline] [CrossRef]

9) Aslaksen PM, Ørbo M, Elvestad R, et al.: Prediction of on-road driving ability after traumatic brain injury and stroke. Eur J Neurol, 2013, 20: 1227-1233. [Medline] [CrossRef]

10) Yamada K, Sasaki T, Kudo A, et al.: Relationships between neuropsychological tests and on-road driving assessment for stroke patients. High Brain Funct Res, 
2013, 33: 270-275 (in Japanese). [CrossRef]

11) Katayama N: Realities and issues of driving restart support in the acute phase. MB Med Reha, 2017, 207: 61-64.

12) Makishita H, Matsunaga K: An error of eye measurement and a change of following distance while driving. Jpn J Ergonomics, 2004, 40: 74-81 (in Japanese). [CrossRef]

13) Barra J, Chauvineau V, Ohlmann T, et al.: Perception of longitudinal body axis in patients with stroke: a pilot study. J Neurol Neurosurg Psychiatry, 2007, 78 : 43-48. [Medline] [CrossRef]

14) Corbetta M, Patel G, Shulman GL: The reorienting system of the human brain: from environment to theory of mind. Neuron, 2008, 58: 306-324. [Medline] [CrossRef]

15) Watanabe S, Takehara I, Hitosugietal M: Cerebral activation patterns of patients operating a driving simulator after brain injury: a functional near-infrared spectroscopy study. JJOMT, 2011, 59: 238-244 (in Japanese).

16) Park MO: A comparison of driving errors in patients with left or right hemispheric lesions after stroke. J Phys Ther Sci, 2015, 27: 3469-3471. [Medline] [CrossRef] 